Historic, Archive Document

Do not assume content reflects current scientific knowledge, policies, or practices. 



\section{COLEMAN GARDENS}

BOX 24

MULTNOMAH, OREGON

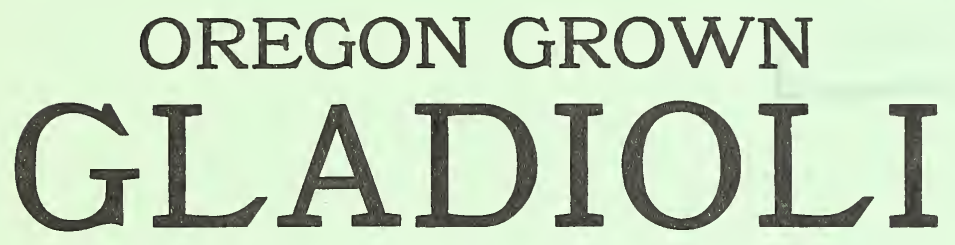

WHOLESALE PRICE LIST

SPRING 1926

Stock quoted subject to prior sale. All varieties true to name.

TRANSPORTATION CHARGES PREPAID on orders over $\$ 3.00$.

TERMS: Cash with order, or $25 \%$ deposit will hold order until March 1st; balance before shipment or C.O.D.

Alice Tiplady (K). Prim., orange saffron . . Prim.....

Anamosa (K). Prim., tall, orange salmon ..........

Anna Eberius (D). Velvety purple ...............

Atlantic. Deep salmon pink, white throat ...........

Bouquet (K). Prim., light yeliow, lower petals penciled pink .............

Byron L. Smith (K). Lavender pink ............

Crimson Glow (Betscher), Crimson ...............

Colors (K). Dark wine maroon, mottled and flaked cream ...............

Charlie Kohler (K). Tall, brilliant scarlet ........

E. J. Shaylor (K). Tall, deep rose-pink .........

Evelyn Kirtland (Austin). Light rose-pink .........

\section{PRICES PER 100 BULBS}

$11 / 2$-up $1 \frac{1 / 4-11 / 2}{1-1} 1 / 4 \quad 3 / 4-1 \quad 1 / 2-3 / 4 \quad 1 / 4-1 / 2 \quad$ B'lts.

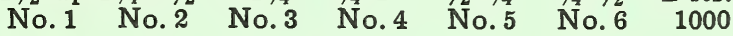

$\begin{array}{lllllll}\$ 4.00 & \$ 3.50 & \$ 2.50 & \ldots & \ldots & \ldots & \$ 1.00\end{array}$

$\begin{array}{lllllll}3.50 & 3.00 & 2.50 & \$ 2.00 & \$ 1.50 & \$ 1.00 & 1.00\end{array}$

$\begin{array}{lllllll}5.00 & 4.00 & 3.00 & 2.00 & \ldots & \ldots & 1.00\end{array}$

$\begin{array}{lllllll}5.00 & 4.50 & 4.00 & 2.75 & 2.00 & 1.50 & 1.50\end{array}$

$\begin{array}{lllllll}\ldots & 3.00 & 2.50 & \ldots & \ldots & \ldots & 1.00\end{array}$

$\begin{array}{llllll}\ldots & 4.00 & 3.00 & 2.50 & 1.50 & 1.00\end{array} \ldots$

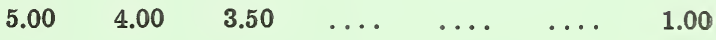

$\begin{array}{lllllll}4.00 & 3.50 & 3.00 & \ldots & \ldots & \ldots & 1.50\end{array}$

$\begin{array}{lllllll}4.50 & 4.00 & 3.00 & \ldots & \ldots & \ldots & 1.50\end{array}$ 4.00 
Fairest White (K). Purest white..$\ldots \ldots \ldots \ldots$. . .

Fayette (K). Giant Salmon rose

Halley (Velthuys). Early, pink ................

Helga (K). Salmon-rose, graceful spike .........

Hereda (Austin). Beautiful mauve ...............

Jack London (D). Light salmon, orange stripes...

Joe Coleman (K). Rich, ruffled-red ............

Le M. Foch (H). Early light-pink .............

Loveliness (Zeestraten). Cream tinted apricot....

Mr. Mark (Velthuys). Light blue .................

Mrs. Dr. Norton (K). Beautiful tall cream and pink

Mrs. Francis King (D). Bright scarlet ..........

Mrs. Frank Pendleton (K). Shell pink, crimsonblotched throat ..........

Mrs. H. E. Bothin (D). Ruffled flesh pink, redblotched throat ................

Mrs. Willard Richardson (D). Deep crimson, maroon center

Muriel (Pfitzer). Light blue, darker throat.....

Mystic (K). Early, deep rose pink .............

1910 Rose (K). Pure rose pink ................

Oak Park (K). Splendid white, slightly marked throat s................

Orange Glory (K). Ruffled, brilliant orange .........

Panama (Banning). Light pink ................

Pride of Lancaster (K). Brilliant orange salmon. .
$1 \frac{1 / 2}{\text {-up } 11 / 4-11 / 2} \quad 1-1 \frac{1 / 4}{3 / 4}-1 \quad 1 / 2-3 / 4 \quad 1 / 4-1 / 2 \quad$ B'lts.

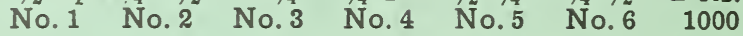

$\begin{array}{lllllll}\$ 6.00 & \$ 5.00 & \$ 4.50 & \$ 3.50 & \$ 3.00 & \$ 2.50 & \$ 2.25\end{array}$

$\begin{array}{lllllll}\ldots & 3.50 & 3.00 & \ldots & \ldots & \ldots & 1.25\end{array}$

$2.00 \quad 1.60 \quad 1.20$

$\begin{array}{lllllll}3.50 & 3.00 & 2.50 & \ldots & \ldots & \ldots & 1.50\end{array}$

$\begin{array}{lllllll}3.00 & 2.50 & 2.00 & \ldots & \ldots & \ldots & 1.00\end{array}$

$5.00 \quad 4.00$

$\begin{array}{lllllll}4.50 & 4.00 & 3.50 & 2.25 & 1.50 & 1.00 & 1.25\end{array}$

$\begin{array}{lllllll}2.00 & 1.50 & 1.25 & 1.00 & \ldots & \ldots & .75\end{array}$

$\begin{array}{lllllll}4.00 & 3.25 & 3.00 & 2.50 & \ldots & \ldots & 1.25\end{array}$

$\begin{array}{llllll}3.00 & 2.40 & 2.00 & \ldots & \ldots & \ldots\end{array} \ldots$

$\begin{array}{llllll}3.50 & 3.00 & 2.00 & \ldots & \ldots & 1.00\end{array}$

$2.00 \quad 1.50 \quad 1.00$

$\begin{array}{lllllll}2.25 & 2.00 & 1.75 & 1.25 & 1.00 & .75 & 1.00\end{array}$

$\begin{array}{llllll}6.00 & 5.00 & \ldots & \ldots & \ldots & 3.50\end{array}$

$\begin{array}{lllllll}6.00 & 5.00 & \ldots & \ldots & \ldots & \ldots & 2.00\end{array}$

$\begin{array}{lllllll}5.00 & 4.00 & 3.25 & 2.50 & \ldots & \ldots & 1.50\end{array}$

$\begin{array}{lllllll}4.50 & 4.00 & 3.50 & 2.25 & \ldots & \ldots & 1.25\end{array}$

$\begin{array}{lllllll}3.00 & 2.50 & 2.00 & 1.50 & 1.00 & \ldots & 1.00\end{array}$

$\begin{array}{lllllll}3.50 & 3.00 & 2.50 & 2.00 & 1.50 & 1.00 & 1.00\end{array}$

$\begin{array}{lllllll}5.00 & 4.50 & 4.00 & \ldots & \ldots & \ldots & 1.50\end{array}$

$2.50 \quad 2.00 \quad 1.50$

6.5

$5.50 \quad 4.50$

3.50

2.00 
Prince of Wales $(\mathrm{H})$. Early salmon pink ...........

Red Amarillas (K). Large brilliant blood-red ......

Red Copper (K). Deep salmon rose, heavily flaked

Red Knight (K). Large, deep ox-blood red, fine. .

Richard Diener (D). Pure rose salmon, fine........

Rose Ash (D). Ashes of

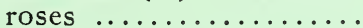

Rose Glory (K). Ruffled, rose pink .............

Rouge Torch (Tracy). Cream white with scarlet throat ................

Scarlano (K). Ruffled, bright orange-red .......

Scarlet Princeps (K). Large scarlet ................

Schwaben (Pfitzer). Large yellow ................

Sweet Lavender (C). Early lavender

Violet Glory (K). Slightly ruffled, violet ..........

War (Groff). Deep brilliant red, tall ................

White Giant $(\mathrm{H})$. Large pure white ............

Wilbrink (Hopman). Early pink

Mixed (Very fine, many Kunderd varieties and seedings)

Bumble Bee and Pink Lily Mixture. Pink .........

E. J. Shaylor and Evelyn Kirtland Mixture. Pink.

Ida Van and Joe Coleman Mixture. Red..........

Mr. Mark and Sentinel Mixture. Light blue and red
$11 / 2$-up $1 \frac{1 / 4-11 / 2}{1-1} 1 \frac{1}{4} \quad 3 / 4-1 \quad 1 / 2-3 / 4 \quad 1 / 4-1 / 2 \quad$ B'lts.

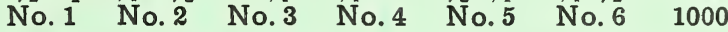

$\begin{array}{lllllll}\$ 3.50 & \$ 3.00 & \$ 2.50 & \$ 1.75 & \ldots & \ldots & \$ 1.00\end{array}$

$\begin{array}{lllllll}\ldots & 6.00 & 5.00 & 4.00 & \ldots & \ldots & 1.50\end{array}$

$\begin{array}{lllllll}\ldots & \ldots & 5.00 & 3.50 & \$ 2.50 & \ldots & 2.00\end{array}$

$\begin{array}{lllllll}5.00 & 4.50 & 4.00 & \ldots & \ldots & \ldots & 2.00\end{array}$

25.00

$\begin{array}{lllllll}4.00 & 3.00 & 2.25 & 1.80 & 1.50 & \$ 1.00 & 1.25\end{array}$

$4.50 \quad 4.00 \quad 3.50$

$\begin{array}{lllllll}2.50 & 2.00 & 1.50 & 1.25 & \ldots & \ldots & 1.00\end{array}$

$\begin{array}{lllllll}5.00 & 4.00 & 3.50 & \ldots & \ldots & \ldots & 1.50\end{array}$

$\begin{array}{lllllll}4.00 & 3.50 & 3.00 & 2.50 & 2.00 & 1.70 & \ldots\end{array}$

$2.20 \quad 1.75 \quad 1.25$

$\begin{array}{lllllll}6.00 & 5.00 & 4.00 & 3.50 & 3.00 & 2.50 & 4.00\end{array}$

$\begin{array}{lllllll}7.00 & 6.00 & \ldots & \ldots & \ldots & \ldots & 3.50\end{array}$

$\begin{array}{lllllll}3.50 & 3.00 & 2.50 & \ldots & \ldots & \ldots & 1.00\end{array}$

7.00

$2.00 \quad 1.50 \quad \ldots . \quad \ldots . \quad .75$

$\begin{array}{lllllll}2.00 & 1.50 & 1.25 & \ldots & \ldots & \ldots & 1.00\end{array}$

$\begin{array}{llll}\cdots & -200 & 1.80 & 1.40\end{array}$

$\begin{array}{llllll}\ldots & \ldots & 1.75 & 1.50 & 1.25 & 1.00\end{array}$

$\begin{array}{llll}2.50 & 2.00 & 1.80 & 1.40\end{array}$

$\begin{array}{llll}2.25 & 2.00 & 1.75 & 1.50\end{array}$

25 or more bulbs at 100 rate 


\section{INTRODUCERS OR ORIGINATORS}

Abbreviations: (K) A. E. Kunderd, (D) Richard Diener, (H) Holland variety, (C) Joe Coleman.

\section{SPECIALS}

Sizes $11 / 4$-up

Each Per10 liant red, mottled........\$ $.25 \$ 2.00$

Henry Ford (D). Purple.. 2.0019 .00

Fern Kyle (K). Large creamy white .......... $\quad .50 \quad 4.50$

Lavender America (K) Pinkish lavender ....... $\quad .50 \quad 4.50$

Maine (Vos) White....... .25 2.00

Mrs. M. S. Burke (D). Apricot and yellow..........

Marshal Foch (K). Large flowers, salmon pink....
$.25 \quad 2.00$

$.25 \quad 2.00$
Purple Glory (K). Giant maroon red ............ $\$ .25 \$ 2.00$

Pythia (K). Giant red..... $.50 \quad 4.50$

Richard Diener (D). Pure rose salmon ............. $.50 \quad 4.50$

Snow Glory (K). Ruffled white, pale lilac throat lines .................. $1.00 \quad 9.00$

Taro (K). American Beauty rose ................. $1.00 \quad 9.00$

Yellow Glory (K). Tall, ruffled yellow, purplish red throat ................. $1.00 \quad 9.00$

Orclers on "SPECIALS" cannot be accepted for less than $\$ 1.00$.

\section{BULBLET PRICES IN QUANTITY}

\begin{tabular}{|c|c|c|c|c|c|}
\hline & Pint & Quart & & Pint & Quart \\
\hline & 4.50 & $\$ 8.50$ & Muriel (Pfitzer) & 2.25 & $\$ 4.00$ \\
\hline & 2.25 & 4.00 & ster $(\mathrm{K}) \ldots$ & 4.50 & 8.50 \\
\hline & 5.25 & 10.00 & & 2.75 & 5.0 \\
\hline & 2.25 & 4.00 & Sweet Lavend & 5.75 & 11.0 \\
\hline rs. Frank Pendleton (K) & 2.25 & 4.00 & War (Groff) & 2.25 & \\
\hline
\end{tabular}

Pints and Quarts are measured by Dry Measure.

Additional Bulbs will be sent for the names and addresses of Quantity Growers.

Subscribe to "The Flower Grower," a first-class floral magazine edited by Madison Cooper, Calcium, New York, and issued monthly. Price $\$ 2.00$ per year, or three years $\$ 4.50$.

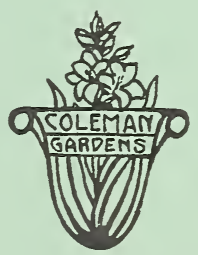

